ARTICLE

Received 1 Sep 2016 | Accepted 9 Feb 2017 | Published 30 Mar $2017 \quad$ DOl: 10.1038/ncomms14933 OPEN

\title{
Temperature-tunable Fano resonance induced by strong coupling between Weyl fermions and phonons in TaAs
}

\author{
B. Xu ${ }^{1,2, \star}$, Y.M. Dai ${ }^{3, \star}$, L.X. Zhao ${ }^{1}$, K. Wang ${ }^{1}$, R. Yang ${ }^{1}$, W. Zhang ${ }^{1}$, J.Y. Liu ${ }^{1}$, H. Xiao ${ }^{2}$, G.F. Chen ${ }^{1,4}$, S.A. Trugman ${ }^{3,5}$, \\ J.-X. Zhu ${ }^{3,5}$, A.J. Taylor ${ }^{6}$, D.A. Yarotski ${ }^{3}$, R.P. Prasankumar ${ }^{3}$ \& X.G. Qiu, ${ }^{1,4}$
}

Strong coupling between discrete phonon and continuous electron-hole pair excitations can induce a pronounced asymmetry in the phonon line shape, known as the Fano resonance. This effect has been observed in various systems. Here we reveal explicit evidence for strong coupling between an infrared-active phonon and electronic transitions near the Weyl points through the observation of a Fano resonance in the Weyl semimetal TaAs. The resulting asymmetry in the phonon line shape, conspicuous at low temperatures, diminishes continuously with increasing temperature. This behaviour originates from the suppression of electronic transitions near the Weyl points due to the decreasing occupation of electronic states below the Fermi level $\left(E_{\mathrm{F}}\right)$ with increasing temperature, as well as Pauli blocking caused by thermally excited electrons above $E_{\mathrm{F}}$. Our findings not only elucidate the mechanism governing the tunable Fano resonance but also open a route for exploring exotic physical phenomena through phonon properties in Weyl semimetals.

\footnotetext{
${ }^{1}$ Beijing National Laboratory for Condensed Matter Physics, Institute of Physics, Chinese Academy of Sciences, P.O. Box 603, Beijing 100190, China. ${ }^{2}$ Center for High Pressure Science and Technology Advanced Research, Beijing 100094, China. ${ }^{3}$ Center for Integrated Nanotechnologies, Los Alamos National Laboratory, Los Alamos, New Mexico 87545, USA. ${ }^{4}$ Collaborative Innovation Center of Quantum Matter, Beijing 100190, China. ${ }^{5}$ Theoretical Division, Los Alamos National Laboratory, Los Alamos, New Mexico 87545, USA. ${ }^{6}$ Associate Directorate for Chemistry, Life and Earth Sciences, Los Alamos National Laboratory, Los Alamos, New Mexico 87545, USA. * These authors contributed equally to this work. Correspondence and requests for materials should be addressed to Y.M.D. (email: ymdai@lanl.gov) or to R.P.P. (email: rpprasan@lanl.gov) or to X.G.Q. (email: xgqiu@iphy.ac.cn).
} 
T he Weyl semimetal (WSM) phase, a novel topological state of quantum matter, has been proposed to exist in materials with two non-degenerate bands crossing at $E_{\mathrm{F}}$ in threedimensional momentum space ${ }^{1}$. At the band crossing points (Weyl points), the electronic dispersion is linear in all three directions, resembling a three-dimensional version of graphene, and the low-energy excitations can be described by Weyl equations, producing a condensed-matter realization of Weyl fermions ${ }^{2}$. Recently, such a WSM state has been discovered in non-centrosymmetric transition-metal monoarsenides and monophosphides (TaAs, TaP, NbAs and $\mathrm{NbP})^{3-8}$, where 12 pairs of Weyl points have been found. Since the Weyl points are located in close proximity to $E_{\mathrm{F}}$ in these materials, interband electronic transitions near the Weyl points occur at a very low energy, $2|\mu|$, where $|\mu|$ represents the chemical potential with respect to the Weyl points ${ }^{9,10}$. This energy scale overlaps optical phonon frequencies ${ }^{11}$. Consequently, strong coupling between electronic transitions near the Weyl points (Weyl fermions) and phonons may arise, manifested through a temperaturetunable Fano resonance.

This is actually a rare occurrence. Fano resonances generally do not occur in conventional metals or semiconductors, since the energy scale of interband electronic transitions in these materials is usually much higher than phonon excitations. However, this interesting phenomenon has been reported in bilayer or few-layer graphene ${ }^{12-14}$, topological insulators ${ }^{15,16}$ and some strongly correlated electron systems, such as stripe-phase nickelates $^{17}$ and high- $T_{\mathrm{c}}$ superconductors ${ }^{18,19}$. The observed Fano resonance in strongly correlated materials is very weak, with $1 / q^{2}$, a parameter that describes the asymmetry of the Fano line shape, only reaching $\sim 0.04$ (refs $17-19$ ). In topological insulators, the effect is also weak $\left(1 / q^{2} \simeq 0.02-0.06\right)$ and cannot be observed without manipulating extrinsic parameters, such as magnetic field ${ }^{15}$, chemical doping ${ }^{16}$ or micro-fabrication on the surface $^{20}$. In contrast, bilayer or few-layer graphene exhibits a strong Fano resonance, with $1 / q^{2} \geq 1$, but the resonance energy is $\sim 1,600 \mathrm{~cm}^{-1}(200 \mathrm{meV})$, much greater than $E_{\mathrm{F}}$, making it impossible to tune the resonance via temperature ${ }^{12-14}$. Moreover, the Fano physics revealed in all the above materials is associated with or has considerable contributions from coupling between phonons and conventional massive fermions.

Here we observe an intrinsic, strong $\left(1 / q^{2} \simeq 1.1\right)$ and temperature-tunable Fano resonance, which arises purely from quantum interference between phonons and massless Weyl fermions, in the recently discovered Weyl semimetal TaAs. We further demonstrate that the Fano line shape can be tuned by changing the occupation of the electronic states near the Weyl points. These observations not only open a novel avenue for exploring exotic quantum phenomena in WSMs, such as the chiral anomaly ${ }^{21-24}$, but also set the stage for a variety of potential applications that take advantage of the ability to tune the Fano resonance using different parameters (for example, temperature, light or magnetic/electric fields).

\section{Results}

Sample growth and characterization. High-quality single crystals of TaAs were synthesized through a chemical vapour transport method ${ }^{23}$. The as-grown crystals are polyhedrons with shiny facets up to $1.5 \mathrm{~mm}$ in size. X-ray diffraction measurements reveal that the as-grown facets are the (001), (107) and (112) surfaces (Supplementary Fig. 1). Systematic optical measurements were carried out on all three surfaces.

Reflectivity and optical conductivity. Figure 1a shows the far-infrared reflectivity $R(\omega)$ measured on the (107) surface of
TaAs at 11 different temperatures from 5 to $300 \mathrm{~K}$ (ref. 10). The relatively high $R(\omega)$ that approaches unity at zero frequency is consistent with the metallic nature of TaAs. A well-defined plasma edge in the far-infrared region suggests very low carrier density, in agreement with the tiny volumes enclosed by the Fermi surfaces in this material ${ }^{3-6}$. In addition to the broad features in $R(\omega)$, a sharp feature can be clearly identified at $\sim 253 \mathrm{~cm}^{-1}(31 \mathrm{meV})$, as indicated by the arrow, which is associated with the infrared-active $A_{1}$ phonon mode (Supplementary Note 1).

In order to gain direct information about this mode, we calculated the optical conductivity $\sigma_{1}(\omega)$ from $R(\omega)$ using a Kramers-Kronig analysis ${ }^{10}$ (more details in Methods section). Figure $1 \mathrm{~b}$ displays $\sigma_{1}(\omega)$ of TaAs on the (107) surface in the far-infrared region at different temperatures. The low-frequency $\sigma_{1}(\omega)$ is dominated by a narrow Drude response alongside prominent linear features, whose origin and temperature dependence have been previously discussed in detail ${ }^{10}$. The $\mathrm{A}_{1}$ mode manifests itself as a sharp peak in the $\sigma_{1}(\omega)$ spectrum, as indicated by the arrow. Figure 1c shows an enlarged view of $\sigma_{1}(\omega)$ in the frequency region of $240-270 \mathrm{~cm}^{-1}$, where the $A_{1}$ mode can be seen more clearly. It is well known that, in the absence of strong electron-phonon coupling, the phonon exhibits a symmetric line shape in $\sigma_{1}(\omega)$ that can be described by a Lorentz oscillator, as schematically illustrated in Fig. 1d. In contrast, strong electron-phonon coupling gives rise to an asymmetric phonon profile in $\sigma_{1}(\omega)$, known as the Fano line shape $\mathrm{e}^{13,14,25}$ (Fig. 1e). As shown in Fig. 1c, the $A_{1}$ mode in TaAs exhibits a striking asymmetric line shape at low temperatures, which is an unequivocal signature of strong electron-phonon coupling. More interestingly, the asymmetry of the phonon line shape diminishes as the temperature rises, suggesting that the coupling-induced Fano resonance in TaAs can be tuned by temperature.

Mechanism governing the temperature-tunable Fano resonance. To quantify the temperature dependence of the $A_{1}$ mode, we extract the phonon line shape by subtracting a linear electronic background in a narrow frequency range at all measured temperatures, as shown in Fig. 2a. At each temperature, the phonon is fit with the Fano line shape ${ }^{25}$,

$$
\sigma_{1}(\omega)=\frac{2 \pi}{Z_{0}} \frac{\Omega^{2}}{\gamma} \frac{q^{2}+\frac{4 q\left(\omega-\omega_{0}\right)}{\gamma}-1}{q^{2}\left(1+\frac{4\left(\omega-\omega_{0}\right)^{2}}{\gamma^{2}}\right)},
$$

where $Z_{0}$ is the vacuum impedance; $\omega_{0}, \gamma$ and $\Omega$ correspond to the resonance frequency, linewidth and strength of the phonon, respectively; $q$ is a dimensionless parameter that describes the asymmetry of the Fano profile. A larger $1 / q^{2}$ indicates more conspicuous asymmetry in the phonon line shape, while for $1 / q^{2}=0$, the symmetric Lorentz line shape is fully recovered. The solid lines in Fig. 2a represent the fitting curves, which describe the measured phonon line shapes reasonably well at all temperatures. This procedure also returns the temperature dependence of the fitting parameters.

Figure $2 \mathrm{~b}$ depicts $1 / q^{2}$ as a function of temperature. While $1 / q^{2}$ adopts a large value of 1.1 at $5 \mathrm{~K}$ (refs $\left.12-14,18\right)$, it decreases dramatically with increasing temperature. This suggests that the $A_{1}$ mode is strongly coupled to a continuum of electron-hole excitations, and the resulting Fano resonance varies significantly with temperature. We first trace the origin of this coupling by examining the band structure of TaAs. Both first-principle calculations and angle-resolved photoemission spectroscopic measurements have revealed 12 pairs of Weyl points in $\mathrm{TaAs}^{3-6}$. These Weyl points are categorized into two types ${ }^{3}$. Four pairs in the $k_{z}=0$ plane, about $2 \mathrm{meV}$ above $E_{\mathrm{F}}$, are defined 
a

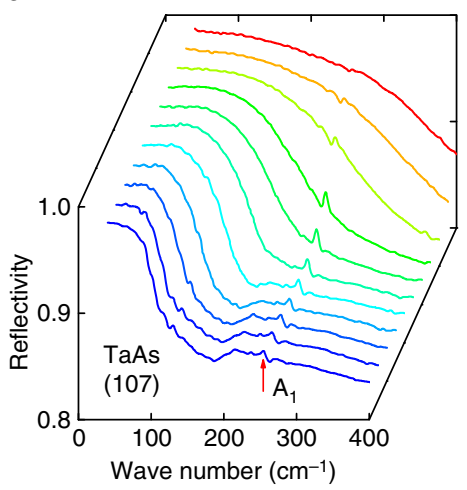

b

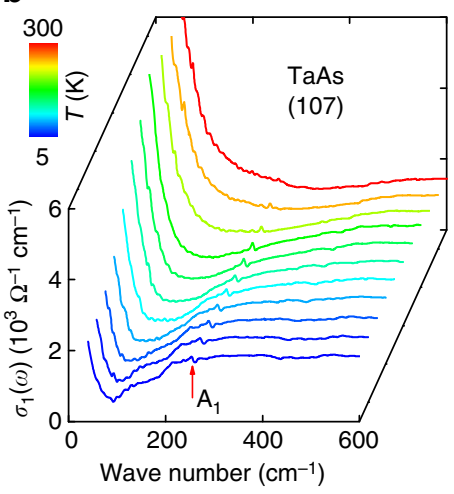

C

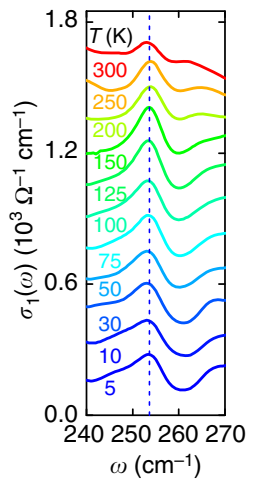

d

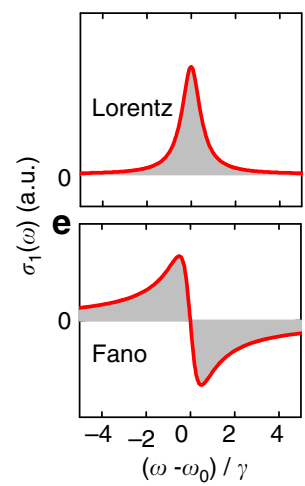

Figure 1 | Reflectivity and optical conductivity of TaAs. (a) Reflectivity of TaAs in the far-infrared region measured at different temperatures on the (107) surface. (b) Optical conductivity of TaAs on the (107) surface up to $600 \mathrm{~cm}^{-1}$ at different temperatures. (c) Enlarged view of the optical conductivity in the region of the infrared-active $A_{1}$ mode at $\sim 253 \mathrm{~cm}^{-1}$. (d) Schematic of the symmetric Lorentz oscillator, which describes the phonon line shape in the optical conductivity without strong electron-phonon coupling. (e) Schematic of the asymmetric Fano resonance, used to describe the phonon line shape in the presence of strong electron-phonon coupling.
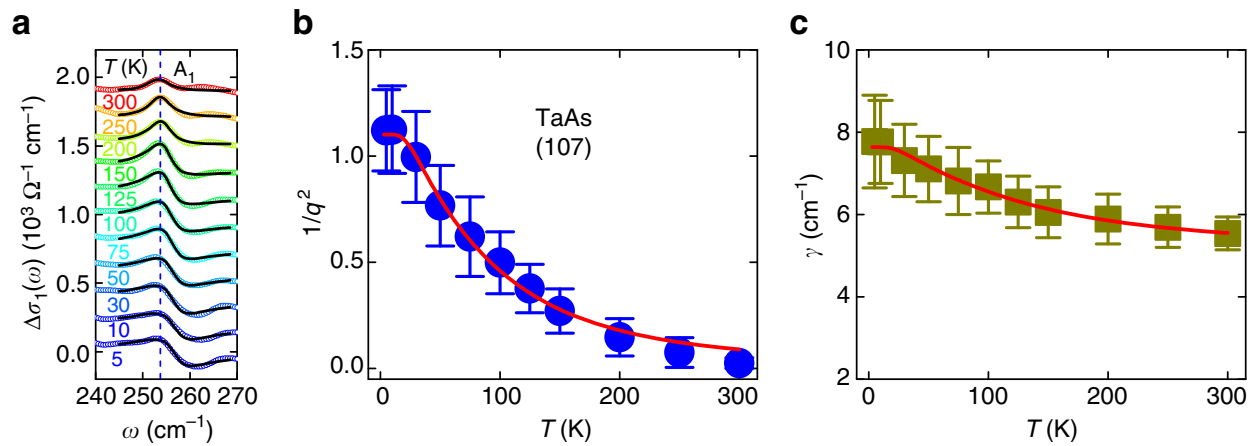

Figure 2 | Fano fit and temperature dependence of fitting parameters. (a) Line shape of the $A_{1}$ phonon, with the electronic background subtracted at different temperatures. The black solid lines through the data denote the Fano fitting results. (b,c) Temperature dependence of the Fano parameter $1 / q^{2}$ and the line width $\gamma$ of the $A_{1}$ mode, respectively. Error bars for both parameters are estimated by fitting the phonon line shape to the Fano equation in different frequency ranges at all measured temperatures. The red solid lines through the data in each panel represent the modelling results.

as W1, while another eight pairs off the $k_{z}=0$ plane, lying about $21 \mathrm{meV}$ below $E_{\mathrm{F}}$, are named W2. Interband electronic transitions in the vicinity of a Weyl point start at $\omega=2|\mu|($ refs 9,10), making it easy to calculate that electronic transitions near W2 turn on at $\omega=42 \mathrm{meV}\left(\sim 336 \mathrm{~cm}^{-1}\right)$. Thus electronic transitions at the frequency of the $A_{1}$ mode $\left(253 \mathrm{~cm}^{-1}\right)$ do not occur near W2. This implies that the $A_{1}$ mode is unlikely to be coupled to the electronic transitions near W2. However, electronic transitions near $\mathrm{W} 1$ set in at $\omega>4 \mathrm{meV}\left(\sim 32 \mathrm{~cm}^{-1}\right)$ and can therefore overlap with the frequency of the $A_{1}$ mode, suggesting that this mode is coupled to the electronic transitions near $\mathrm{W} 1$.

Having attributed the asymmetric line shape of the $A_{1}$ mode to its strong coupling with the electronic transitions near W1, we proceed to understand the temperature dependence of this mode. In WSMs, since the Weyl points are in close proximity to $E_{\mathrm{F}}$, electronic transitions near the Weyl points can be dramatically affected by thermal excitations, thus changing the line shape of the phonon that is coupled to these transitions. Figure 3 shows the band structure along three momentum directions near W1 in TaAs. The occupation probabilities of the electronic states in these bands (colour maps) are calculated using the Fermi-Dirac distribution function at three different temperatures $(5,150$ and $300 \mathrm{~K})$, from which we see that the occupation of the electronic states near W1 depends strongly on the temperature. At $5 \mathrm{~K}$ (Fig. $3 \mathrm{a}-\mathrm{c}$ ), the electronic states below $E_{\mathrm{F}}$ are fully occupied (blue), while the states above $E_{\mathrm{F}}$ are empty (white). In this case, interband transitions at the energy of the $A_{1}$ mode $\hbar \omega_{0}$ are strong, as indicated by the thick arrows. As the temperature increases from 5 to $300 \mathrm{~K}$, thermal excitations cause vacant states to appear below $E_{\mathrm{F}}$ and electronic states above $E_{\mathrm{F}}$ to be partially occupied. Electronic transitions at $\hbar \omega_{0}$ are significantly suppressed (illustrated by the thin arrows in Fig. 3), because the available initial states for these transitions decrease, and many of the final states are Pauli blocked by thermally excited electrons. This suppression of the electronic transitions near W1 is directly responsible for the change in the $A_{1}$ phonon line shape.

For a more quantitative analysis, the dimensionless parameter $q$ in the Fano theory is given by 13,25

$$
q=\frac{1}{\pi V_{\mathrm{e}-\mathrm{ph}} D_{\mathrm{e}-\mathrm{h}}\left(\omega_{0}, T\right)} \times \frac{\mu_{\mathrm{ph}}}{\mu_{\mathrm{e}-\mathrm{h}}},
$$

where $V_{\mathrm{e}-\mathrm{ph}}$ is the electron-phonon coupling strength; $D_{\mathrm{e}-\mathrm{h}}\left(\omega_{0}, T\right)$ is the joint electron-hole pair density of states at the frequency of the $A_{1}$ mode $\omega_{0}$ for a given temperature $T$; and $\mu_{\mathrm{ph}}$ and $\mu_{\mathrm{e}-\mathrm{h}}$ represent the optical matrix elements for phonon and electron-hole pair excitations, respectively. In this equation, we note that raising $T$ mainly modifies $D_{\mathrm{e}-\mathrm{h}}\left(\omega_{0}, T\right)$ by thermally 


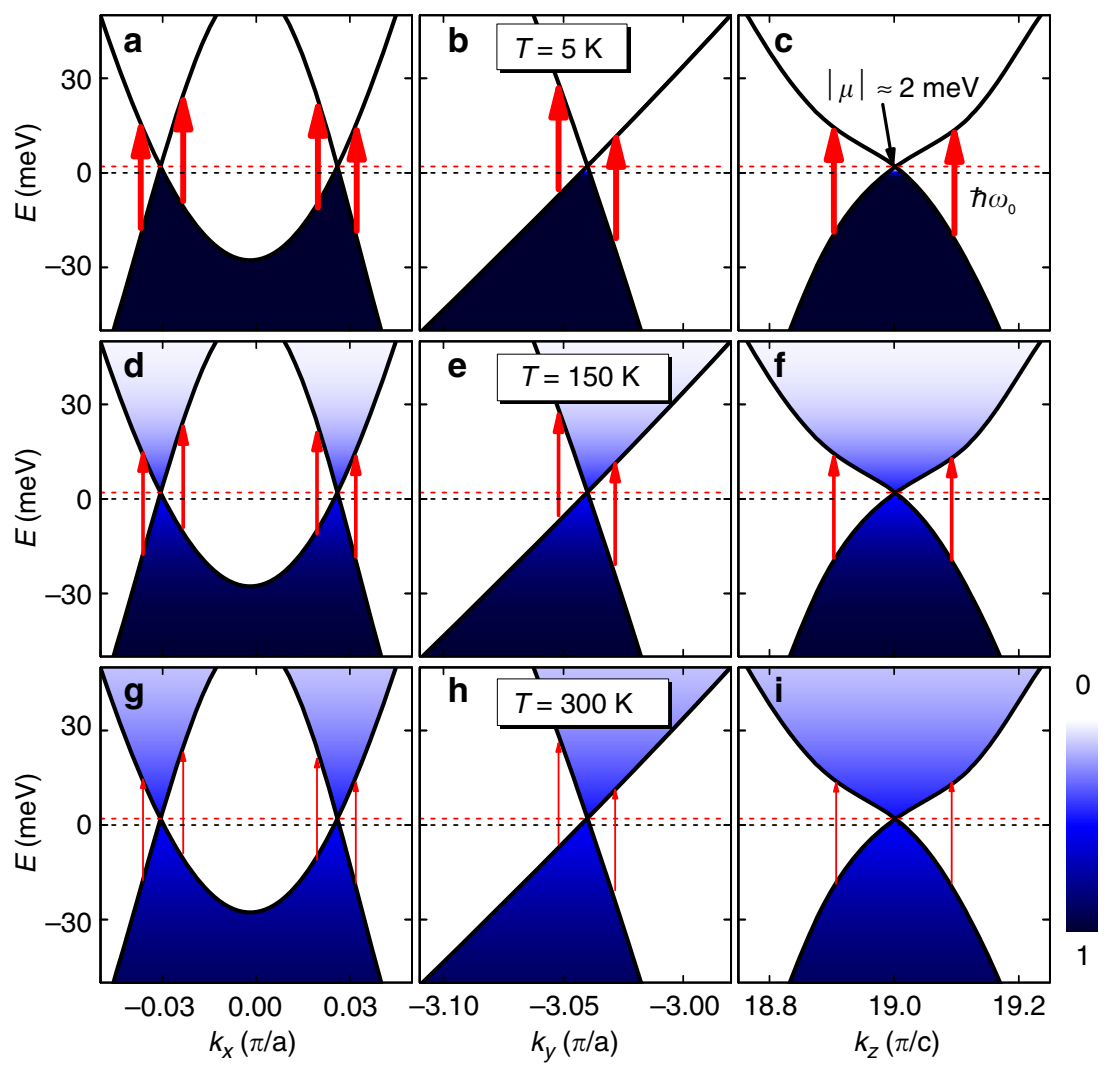

Figure 3 | Occupation probability of the electronic states near W1. Band structure along three different momentum directions near the Weyl points W1 in TaAs. The black and red dashed lines in each panel correspond to the Fermi level and the energy of W1 $(|\mu| \simeq 2$ meV), respectively. The colour maps, which are calculated from the Fermi-Dirac distribution function $f(E)$, denote the occupation probability of the electronic states at different temperatures of $5 \mathrm{~K}(\mathbf{a}-\mathbf{c}), 150 \mathrm{~K}(\mathbf{d}-\mathbf{f})$ and $300 \mathrm{~K}(\mathbf{g}-\mathbf{i})$. The red arrows represent the electronic transitions at the energy of the $A_{1}$ mode $\hbar \omega_{0}$. The thickness of each arrow schematically depicts the transition amplitude.

exciting electrons to the electronic states above $E_{\mathrm{F}}$ and creating holes below $E_{\mathrm{F}}$. Near the Weyl points, the finite-temperature joint electron-hole pair density of states at $\hbar \omega_{0}$ takes the form

$$
D_{\mathrm{e}-\mathrm{h}}\left(\omega_{0}, T\right)=D_{\mathrm{e}-\mathrm{h}}^{0}\left(\omega_{0}\right)\left[f\left(-\frac{\hbar \omega_{0}}{2}\right)-f\left(\frac{\hbar \omega_{0}}{2}\right)\right],
$$

where $f(\epsilon)=1 /\left[\mathrm{e}^{(\epsilon-\mu) / k_{\mathrm{B}} T}+1\right]$ is the Fermi function, with $\epsilon$ representing the energy of the single-particle state with respect to the Weyl points and $D_{\mathrm{e}-\mathrm{h}}^{0}\left(\omega_{0}\right)$ being the zerotemperature joint electron-hole pair density of states at $\hbar \omega_{0}$. The red solid curve in Fig. $2 b$ is the least-squares fit to the experimental temperature dependence of $1 / q^{2}$ (blue solid circles) using equation (2). The excellent agreement between our experimental data and the model further underlines the intimate link between the line shape of the $A_{1}$ mode and the electronic transitions near $\mathrm{W} 1$, which are continuously suppressed with increasing temperature due to the reduced occupation of the electronic states below $E_{\mathrm{F}}$ and Pauli blocking from thermally excited electrons above $E_{\mathrm{F}}$.

A careful examination of the temperature dependence of the $\mathrm{A}_{1}$ phonon linewidth $\gamma$ (Fig. 2c) leads us to the same conclusion. In the case of weak electron-phonon coupling, phonon decay is dominated by the anharmonic effect: a zone-centre phonon decays into two acoustic modes with the same frequencies and opposite momenta ${ }^{26,27}$. The temperature dependence of the phonon linewidth $\gamma^{\mathrm{ph}-\mathrm{ph}}(T)$ for this process follows

$$
\gamma^{\mathrm{ph}-\mathrm{ph}}(T)=\gamma_{0}^{\mathrm{ph}-\mathrm{ph}}\left(1+\frac{2}{\mathrm{e}^{\frac{\hbar \omega_{0}}{2 \mathrm{~B}_{\mathrm{B}} T}}-1}\right),
$$

where $\gamma^{\mathrm{ph}-\mathrm{ph}}$ is the residual linewidth at zero temperature. Apparently, this model does not account for the behaviour of the $\mathrm{A}_{1}$ mode in TaAs, since it gives an increasing $\gamma$ as the temperature is raised, which is opposite to our experimental observation. Instead, strong electron-phonon coupling must be taken into account to understand the temperature dependence of $\gamma$ in TaAs. In a system with strong electron-phonon coupling, a phonon can also decay by creating an electron-hole pair ${ }^{28}$. This process is sensitive to $D_{\mathrm{e}-\mathrm{h}}\left(\omega_{0}, T\right)$ and is thus suppressed with increasing temperature due to thermal excitations, resulting in a temperature-dependent phonon linewidth $\gamma^{\mathrm{e}-\mathrm{ph}}(T)$

$$
\gamma^{\mathrm{e}-\mathrm{ph}}(T)=\gamma_{0}^{\mathrm{e}-\mathrm{ph}}\left[f\left(-\frac{\hbar \omega_{0}}{2}\right)-f\left(\frac{\hbar \omega_{0}}{2}\right)\right],
$$

where $\gamma_{0}^{\mathrm{e}-\mathrm{ph}}$ represents a residual linewidth. Consequently, the temperature-dependent linewidth of a phonon mode that is strongly coupled to electronic excitations is given by $\gamma(T)=\gamma^{\mathrm{ph}-\mathrm{ph}}(T)+\gamma^{\mathrm{e}-\mathrm{ph}}(T)$. This equation gives an excellent description to the measured temperature dependence of the linewidth for the $A_{1}$ mode in TaAs, as shown by the red solid curve in Fig. 2c. The above observations explicitly demonstrate that the $A_{1}$ mode is strongly coupled to the electronic transitions near W1, and both the line shape and linewidth of this phonon are closely tied to these transitions, which can be 
continuously tuned by temperature through varying the occupation of the electronic states near W1.

To lend further credence to our experimental results and analysis, we performed optical measurements at 12 different temperatures on the (112) surface. Essentially identical behaviour was revealed for the $A_{1}$ mode (Supplementary Fig. 3). We then utilized the same methods and models to analyse the line shape and linewidth of this mode observed on the (112) surface (Supplementary Fig. 4), reaching the same conclusions.

Finally, we note that although electronic transitions at $\hbar \omega_{0}$ are absent near W2 at low temperatures, Fermi smearing at high temperatures may relax the Pauli blocking, allowing these transitions to occur. From equations ( 2 and 3 ), we can easily calculate the asymmetry of the $A_{1}$ mode arising from coupling to $\mathrm{W} 2$ at $300 \mathrm{~K}: 1 / q_{\mathrm{W} 2}^{2}(300 \mathrm{~K}) \simeq 0.07$. This small value of $1 / q_{\mathrm{W} 2}^{2}$, which vanishes quickly with decreasing temperature, suggests that electronic transitions near W2 do not give rise to a noticeable Fano resonance in the $A_{1}$ mode of TaAs over the measured temperature range (5-300 K). However, the enhancement of these transitions with increasing temperature may produce a strong Fano resonance at high enough temperatures. It is also worth pointing out that changing the occupation of the electronic states near W1 via other methods, such as electrical gating or femtosecond optical excitation, should induce a similar change in the Fano line shape of the $A_{1}$ mode in TaAs.

\section{Discussion}

The strong coupling between Weyl fermions and phonons enables the study of exotic quantum phenomena in WSMs by tracking the properties of phonons. One interesting proposal derived from our findings is to provide experimental evidence for the chiral anomaly ${ }^{21-24}$, in which the application of parallel electric (E) and magnetic (B) fields pumps electrons from one Weyl point to the other with opposite chirality at a rate proportional to $\mathbf{E} \cdot \mathbf{B}$, leading to a shift of $E_{\mathrm{F}}$ in opposite directions at different Weyl points. This $E_{\mathrm{F}}$ shift caused by the chiral anomaly can significantly affect the electronic transitions near the Weyl points, which accordingly changes the line shape of the phonon that is coupled to these transitions.

For more insight, we can calculate the chiral anomaly-induced $E_{\mathrm{F}}$ shift, given by,29

$$
\Delta E_{\mathrm{F}}=\left(\frac{3 \mathrm{e}^{2} \hbar v_{\mathrm{F}}^{3} \tau}{2} \mathbf{E} \cdot \mathbf{B}\right)^{1 / 3}
$$

where $v_{\mathrm{F}}$ is the Fermi velocity and $\tau$ is the scattering time between different Weyl points. To obtain a realistic estimate, we take $v_{\mathrm{F}}=5 \times 10^{5} \mathrm{~m} \mathrm{~s}^{-1}\left(\right.$ refs 6,23,30), $\tau=5 \times 10^{-11}$ s (refs 9,24,29), $\mathbf{B}=5 \mathrm{~T}$ and $\mathbf{E}=1 \mathrm{~V} \mathrm{~mm}^{-1}$, giving $\Delta E_{\mathrm{F}}=31.34 \mathrm{meV}$. Such an $E_{\mathrm{F}}$ shift would completely block the electronic transitions at $\hbar \omega_{0}$ near W1 (Fig. 3), resulting in a full recovery of the symmetric Lorentz line shape. This theoretical estimate thus suggests that, at low temperatures, the striking asymmetry of the $A_{1}$ phonon line shape in TaAs should decrease with increasing $\mathbf{E} \cdot \mathbf{B}$, due to the chiral anomaly-induced $E_{\mathrm{F}}$ shift, until a symmetric Lorentz line shape is fully recovered at high E-B fields. Experimental observation of this effect would be a strong evidence for the chiral anomaly in WSMs.

Furthermore, our observations suggest a variety of potential device applications. The Fano resonance in optical spectra arises from strong quantum interference between topologically non-trivial Weyl fermions and phonons, and the properties of Weyl fermions can be manipulated by a variety of different parameters, such as temperature, light ${ }^{31,32}$ and electric/magnetic fields ${ }^{23,24}$. This could thus lead to a new class of devices operating at the Fano resonance that could be controlled by any one of these parameters, making them unusually flexible and thus potentially useful in a wide range of applications.

In conclusion, we have found compelling evidence for strong coupling between the infrared-active $A_{1}$ phonon mode and electronic transitions near W1 (Weyl fermions) in TaAs through the observation of a Fano resonance. Varying the temperature, which changes the occupation of the electronic states in proximity to $E_{\mathrm{F}}$, can continuously tune the amplitude of the electronic transitions near W1, thus manipulating the line shape of the $\mathrm{A}_{1}$ mode. Our findings not only suggest a new approach for investigating exotic quantum phenomena in WSMs, such as the chiral anomaly, through the behaviour of phonons, but also imply a broad range of potential applications, such as in thermo-, magneto- and electro-optical devices. Please note that, after our manuscript was submitted, we noticed two preprints (refs 33,34 ) that theoretically propose detecting the chiral anomaly in WSMs through phonon dynamics.

\section{Methods}

Sample synthesis. High-quality TaAs single crystals were grown through a chemical vapour transport method ${ }^{23}$. A previously reacted polycrystalline TaAs was filled in a quartz ampoule using iodine $\left(2 \mathrm{mg} \mathrm{cm}^{-3}\right)$ as the transporting agent. After evacuating and sealing, the ampoule was kept at the growth temperature for 3 weeks. Large polyhedral crystals with dimensions up to $1.5 \mathrm{~mm}$ are obtained in a temperature field of $\Delta T=1,150-1,000^{\circ} \mathrm{C}$.

Optical measurements. Near normal incident reflectivity $\mathrm{R}(\omega)$ was measured over a broad frequency range on a Bruker VERTEX 80v FTIR spectrometer. In order to accurately measure the absolute $R(\omega)$ of the samples, an in situ gold overcoating technique ${ }^{35}$ was used. $\mathrm{R}(\omega)$ from 40 to $15,000 \mathrm{~cm}^{-1}$ were measured at 11 different temperatures from 300 down to $5 \mathrm{~K}$ on three different surfaces of as-grown single crystals in an ARS-Helitran cryostat. Since a broad spectral range is required for a Kramers-Kronig analysis, we extended our data to the ultraviolet range (up to 50,000 $\mathrm{cm}^{-1}$ ) at room temperature using an AvaSpec-2048 $\times 14$ fiber optic spectrometer.

Kramers-Kronig analysis. The real part of the optical conductivity $\sigma_{1}(\omega)$ has been determined via a Kramers-Kronig analysis of $R(\omega)^{36}$. Below the lowest measured frequency, we used a Hagen-Rubens $(R=1-A \sqrt{\omega})$ form for the low-frequency extrapolation. Above the highest measured frequency, we assumed a constant reflectivity up to $12.5 \mathrm{eV}$, followed by a free-electron $\left(\omega^{-4}\right)$ response.

Data availability. All data that support the findings of this study are available from the corresponding authors upon request.

\section{References}

1. Wan, X., Turner, A. M., Vishwanath, A. \& Savrasov, S. Y. Topological semimetal and fermi-arc surface states in the electronic structure of pyrochlore iridates. Phys. Rev. B 83, 205101 (2011).

2. Weyl, H. Electron and gravitation. I. Z. Phys. 56, 330-352 (1929).

3. Weng, H., Fang, C., Fang, Z., Bernevig, B. A. \& Dai, X. Weyl semimetal phase in noncentrosymmetric transition-metal monophosphides. Phys. Rev. X 5, 011029 (2015).

4. Huang, S.-M. et al. A Weyl Fermion semimetal with surface Fermi arcs in the transition metal monopnictide TaAs class. Nat. Commun. 6, 7373 (2015).

5. Xu, S.-Y. et al. Discovery of a Weyl fermion semimetal and topological Fermi arcs. Science 349, 613-617 (2015).

6. Lv, B. Q. et al. Observation of Weyl nodes in TaAs. Nat. Phys. 11, 724-727 (2015).

7. Lv, B. Q. et al. Experimental discovery of Weyl semimetal TaAs. Phys. Rev. X 5, 031013 (2015).

8. Yang, L. X. et al. Weyl semimetal phase in the non-centrosymmetric compound TaAs. Nat. Phys. 11, 728-732 (2015).

9. Ashby, P. E. C. \& Carbotte, J. P. Chiral anomaly and optical absorption in Weyl semimetals. Phys. Rev. B 89, 245121 (2014).

10. Xu, B. et al. Optical spectroscopy of the Weyl semimetal TaAs. Phys. Rev. B 93, 121110 (2016).

11. Liu, H. W. et al. Raman study of lattice dynamics in the Weyl semimetal TaAs. Phys. Rev. B 92, 064302 (2015).

12. Kuzmenko, A. B. et al. Gate tunable infrared phonon anomalies in bilayer graphene. Phys. Rev. Lett. 103, 116804 (2009). 
13. Tang, T.-T. et al. A tunable phonon-exciton Fano system in bilayer graphene. Nat. Nano 5, 32-36 (2010).

14. Li, Z. et al. Structure-dependent Fano resonances in the infrared spectra of phonons in few-layer graphene. Phys. Rev. Lett. 108, 156801 (2012).

15. LaForge, A. D. et al. Optical characterization of $\mathrm{Bi}_{2} \mathrm{Se}_{3}$ in a magnetic field: Infrared evidence for magnetoelectric coupling in a topological insulator material. Phys. Rev. B 81, 125120 (2010).

16. Sim, S. et al. Tunable Fano quantum-interference dynamics using a topological phase transition in $\left(\mathrm{Bi}_{1-x} \mathrm{In}_{x}\right)_{2} \mathrm{Se}_{3}$. Phys. Rev. B 91, 235438 (2015).

17. Coslovich, G. et al. Ultrafast charge localization in a stripe-phase nickelate. Nat. Commun. 4, 2643 (2013).

18. $\mathrm{Xu}, \mathrm{B}$. et al. Anomalous phonon redshift in $\mathrm{K}$-doped $\mathrm{BaFe}_{2} \mathrm{As}_{2}$ iron pnictides. Phys. Rev. B 91, 104510 (2015).

19. Homes, C. C., Dai, Y. M., Schneeloch, J., Zhong, R. D. \& Gu, G. D. Phonon anomalies in some iron telluride materials. Phys. Rev. B 93, 125135 (2016).

20. Autore, M. et al. Plasmon-phonon interactions in topological insulator microrings. Adv. Opt. Mater. 3, 1257-1263 (2015).

21. Nielsen, H. \& Ninomiya, M. The Adler-Bell-Jackiw anomaly and Weyl fermions in a crystal. Phys. Lett. B 130, 389-396 (1983).

22. Hosur, P. \& Qi, X. Recent developments in transport phenomena in Weyl semimetals. Comptes Rendus Physique 14, 857-870 (2013).

23. Huang, X. et al. Observation of the chiral-anomaly-induced negative magnetoresistance in 3D Weyl semimetal TaAs. Phys. Rev. X 5, 031023 (2015).

24. Zhang, C.-L. et al. Signatures of the Adler-Bell-Jackiw chiral anomaly in a Weyl fermion semimetal. Nat. Commun. 7, 10735 (2016).

25. Fano, U. Effects of configuration interaction on intensities and phase shifts. Phys. Rev. 124, 1866-1878 (1961).

26. Klemens, P. G. Anharmonic decay of optical phonons. Phys. Rev. 148, 845-848 (1966)

27. Menéndez, J. \& Cardona, M. Temperature dependence of the first-order Raman scattering by phonons in Si, Ge, and $\alpha$-Sn: anharmonic effects. Phys. Rev. B 29, 2051-2059 (1984)

28. Bonini, N., Lazzeri, M., Marzari, N. \& Mauri, F. Phonon anharmonicities in graphite and graphene. Phys. Rev. Lett. 99, 176802 (2007).

29. Hosur, P. \& Qi, X.-L. Tunable circular dichroism due to the chiral anomaly in Weyl semimetals. Phys. Rev. B 91, 081106 (2015).

30. Luo, Y. et al. Electron-hole compensation effect between topologically trivial electrons and nontrivial holes in NbAs. Phys. Rev. B 92, 205134 (2015).

31. Chan, C.-K., Oh, Y.-T., Han, J. H. \& Lee, P. A. Type-II Weyl cone transitions in driven semimetals. Phys. Rev. B 94, 121106 (2016).

32. Hübener, H., Sentef, M. A., De Giovannini, U., Kemper, A. F. \& Rubio, A. Creating stable Floquet-Weyl semimetals by laser-driving of 3D Dirac materials. Nat. Commun. 8, 13940 (2017).

33. Song, Z., Zhao, J., Fang, Z. \& Dai, X. Detecting chiral magnetic effect by lattice dynamics. Preprint at https://arxiv.org/abs/1609.05442 (2016).

34. Rinkel, P., Lopes, P. L. S. \& Garate, I. Signatures of the chiral anomaly in phonon dynamics. Preprint at https://arxiv.org/abs/1610.03073 (2016).
35. Homes, C. C., Reedyk, M., Cradles, D. A. \& Timusk, T. Technique for measuring the reflectance of irregular, submillimeter-sized samples. Appl. Opt. 32, 2976-2983 (1993).

36. Dressel, M. \& Grüner, G. Electrodynamics of Solids (Cambridge University press, 2002).

\section{Acknowledgements}

We thank Ricardo Lobo, Yongkang Luo, Simin Nie and Hongming Weng for illuminating discussions. Work at IOP CAS was supported by MOST (973 Project Nos. 2015CB921303 and 2015CB921102) and NSFC (Grant Nos. 91121004, 91421304 and 11374345). Work at LANL was performed at the Center for Integrated Nanotechnologies, a US Department of Energy, Office of Basic Energy Sciences user facility, and funded by the LANL LDRD program and by the UC Office of the President under the UC Lab Fees Research Program, Grant ID No. 237789. H.X. is supported by NSFC, Grant No. U1530402.

\section{Author contributions}

B.X. carried out the optical measurements with the assistance of K.W., R.Y., W.Z. and J.Y.L.; L.X.Z. and G.F.C. synthesized the single crystals; B.X., Y.M.D., H.X., A.J.T., D.A.Y., R.P.P. and X.G.Q. analysed the data; S.A.T. and J.-X.Z. contributed to theoretical models Y.M.D. wrote the manuscript; all authors made comments on the manuscript; R.P.P. and X.G.Q. supervised the project.

\section{Additional information}

Supplementary Information accompanies this paper at http://www.nature.com/ naturecommunications

Competing interests: The authors declare no competing financial interests.

Reprints and permission information is available online at http://npg.nature.com/ reprintsandpermissions/

How to cite this article: $\mathrm{Xu}, \mathrm{B}$. et al. Temperature-tunable Fano resonance induced by strong coupling between Weyl fermions and phonons in TaAs. Nat. Commun. 8, 14933 doi: 10.1038/ncomms14933 (2017)

Publisher's note: Springer Nature remains neutral with regard to jurisdictional claims in published maps and institutional affiliations.

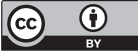

This work is licensed under a Creative Commons Attribution 4.0 International License. The images or other third party material in this article are included in the article's Creative Commons license, unless indicated otherwise in the credit line; if the material is not included under the Creative Commons license, users will need to obtain permission from the license holder to reproduce the material. To view a copy of this license, visit http://creativecommons.org/licenses/by/4.0/

(C) The Author(s) 2017 